\title{
Correction of Traumatic Ankle Valgus and Procurvatum using the Taylor Spatial Frame: A Case Report
}

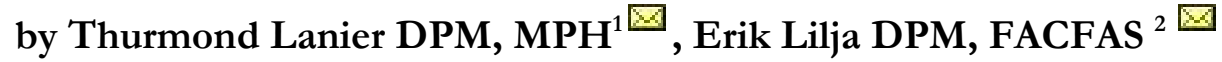

\section{The Foot and Ankle Online Journal 4 (5): 3}

Injuries about the ankle joint can have devastating consequences when left untreated or undertreated, and treatment is especially important in the pediatric population. Physeal injury may occur that can result in abnormal growth patterns. External fixation can be used to correct ankle and tibial deformities, and the Taylor Spatial Frame (TSF) can be used to more easily correct triplane deformities. A case study is presented to demonstrate the use of the TSF in correction of ankle valgus and tibial procurvatum.

Key words: Epiphysis, Ilizarov frame, External fixation, CORA

Published: May, 2011

This is an Open Access article distributed under the terms of the Creative Commons Attribution License. It permits unrestricted use, distribution, and reproduction in any medium, provided the original work is properly cited. (The Foot and Ankle Online Journal (www.faoj.org), 2011 All rights reserved.

$\mathrm{I}$ njuries about the ankle joint such as fractures, chronic ligamentous injuries, and osteochondral lesions can result in post-traumatic arthritis. These injuries are more devastating when they are left untreated or undertreated. When injuries of this nature happen in the pediatric population, special considerations must be taken to preserve physeal growth plates and prevent the occurrence of future growth disturbances. When a disturbance does occur at the epiphysis, depending how severe the deformity, surgical correction may be indicated. Internal fixation may be used to accomplish correction, but external fixation may be preferred if the deformity is severe which may result in soft tissue compromise if corrected acutely.

Address correspondence to: Thurmond Lanier, DPM, MPH, Swedish Medical Center, 747 Broadway, Seattle, WA, 98122.

\footnotetext{
${ }^{1}$ PGY 2, Swedish Medical Center, 747 Broadway, Seattle, WA, 98122 2 Attending physician, Swedish medical center, 747 Broadway, Seattle, WA, 98122, Private practice, 9501 5th Ave. NE, Seattle, WA, 98115.
}

Ilizarov circular frames have had widespread use in the correction of angular deformities. ${ }^{1}$ With more complex deformities (such as those in multiple planes), Ilizarov frames may be used but the application of hinges are needed and more complex pre-operative planning as well as more intricate postoperative manipulation is needed. With the advent of the Taylor Spatial Frame (TSF), deformities can be reduced in all three planes simultaneously. The TSF has two rings and six struts that are expandable. ${ }^{2}$ The deformity parameters are measured by the surgeon and are introduced into the TSF software in which a prescription is printed. ${ }^{3}$ We present a case report of a pediatric case where a TSF was utilized for correction of a multiplanar ankle deformity. 

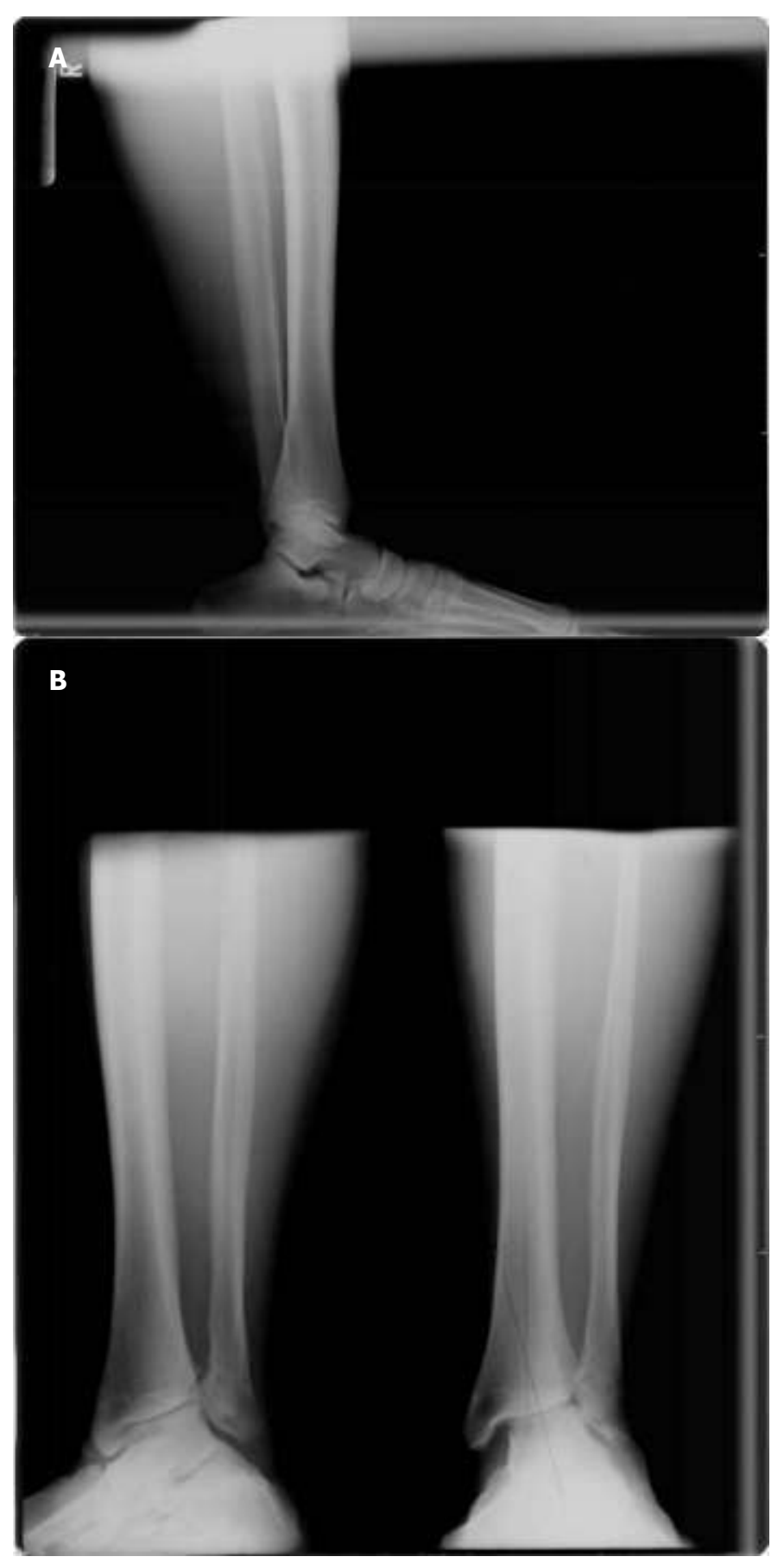

Figures 1A and 1B Lateral pre-operative radiograph displaying tibial procurvatum and relatively narrow joint space. (A) The anterior posterior (AP) and mortise preoperative radiographs demonstrating significant valgus of the mortise and increased medial clear space. Also note decreased tibia-fibula overlap. (B)

\section{Case Report}

A 14 year-old patient presented to clinic with complaints of right ankle pain. The patient related having a history of a right ankle fracture which happened 4 years prior. The patient states that he was making a jump on his quad and ended up catching the right foot on the top of the rear tire and felt a snap. The patient was diagnosed with an ankle fracture and was treated conservatively in a cast for approximately six weeks. The patient removed his own cast at that time and began weight bearing. The patient continued to have lateral ankle pain and noticed that his foot was in an abnormal position relative to his ankle. Radiographs were obtained 2 years after the initial injury which showed collapse of the lateral portion of the epiphysis and shortening with lateral displacement of the fibula with an increase in medial clear space. (Figs. 1A and 1B) This also resulted in valgus position of the ankle joint. The patient was given conservative treatment for approximately two years until his growth plates were closed and operative intervention was then initiated.

\section{Operative Technique/Post-operative care}

The TSF consisted of two tibial rings connected by six struts. The proximal ring was applied to the tibia via two half pins. The half pins were applied perpendicular to the posterior crest of the tibia and confirmed with fluoroscopy. The distal ring was placed with two wires and a half pin and was placed distal to where the proposed osteotomy would be. The distal ring was placed in relative malalignment with the distal tibia so when the deformity was corrected the frame would be in a neutral position. An incision was then made along the anteromedial aspect of the tibia. Layered dissection was taken down to the tibia shaft and a through and through osteotomy was made with a sagittal saw. The osteotomy was made as distal in the tibia shaft as possible as the center of rotation of angulation (CORA) was located within the ankle joint. The CORA represents the apex of the deformity and in most cases is the optimal location to place the osteotomy. 


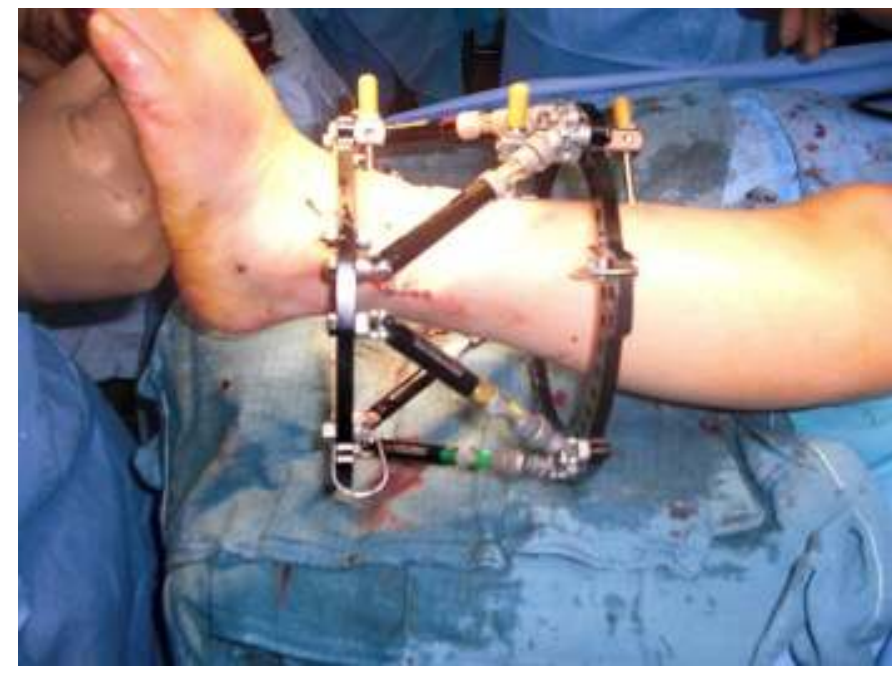

Figure 2 Intra-operative image showing application of the Taylor Spatial Frame (TSF).

If the CORA is located within a joint, generally the osteotomy is made proximal to the CORA within bone with good blood supply. A lateral incision was then made over the fibula with dissection taken down to the shaft. Utilizing a sagittal saw a transverse osteotomy was made. The final frame construction was made sure to be orthogonal to the tibia. (Fig. 2) Post-operatives radiographs were taken to ensure proper alignment of the frame. (Figs. 3 and 4) Adjustments were started approximately 10 days after surgery. Radiographs were taken on a weekly basis. The only complication that occurred was a pin tract infection which resolved with antibiotics. The frame was removed in 3 months when bony consolidation of the osteotomy was identified on radiographs. (Figs. 5 and 6)

\section{Discussion}

The use of the TSF has been described in numerous cases in the literature. The frame has been utilized in accomplishing ankle arthrodesis in patients with and without ankle and tibial deformities. Thiryayi, et al., described ankle fusion using the TSF in 10 patients. ${ }^{2}$ The patients stayed in the fixator for an average of 24 weeks.

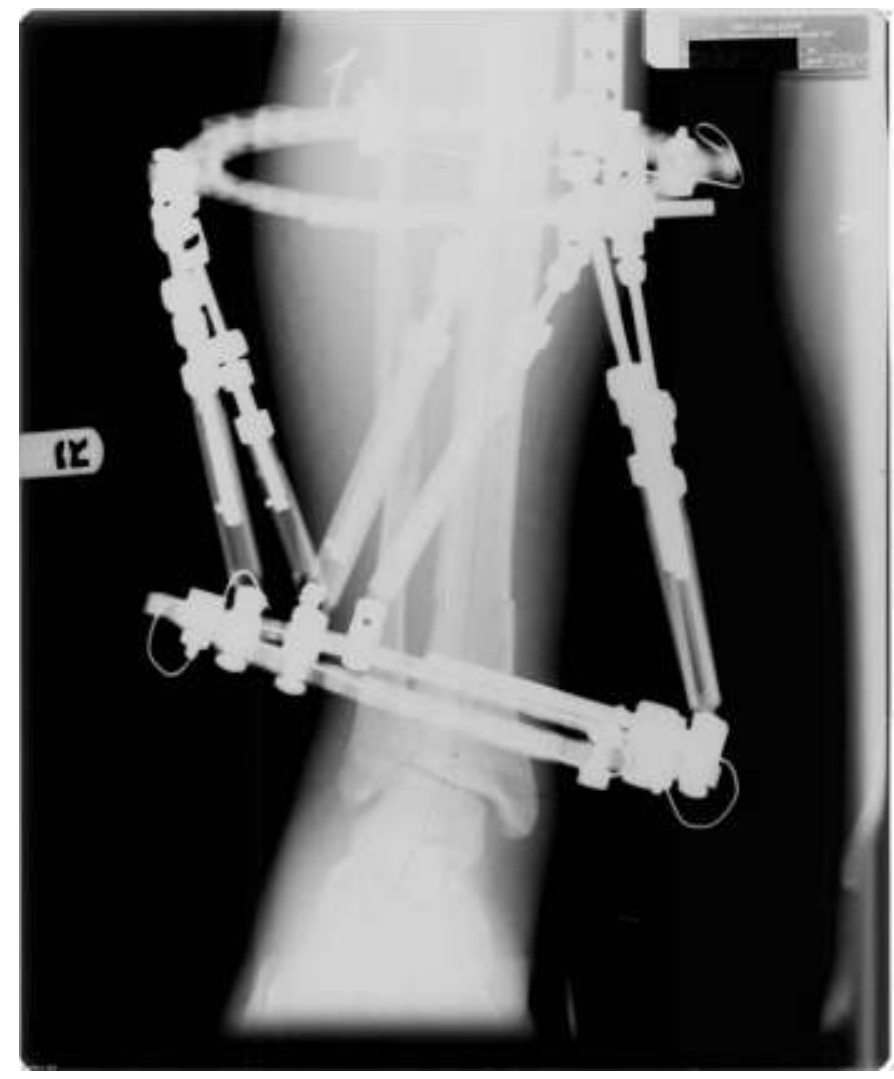

Figure 3 Immediate AP post-operative radiograph. Note distal ring with built-in deformity to match deformity at ankle joint.

All patients demonstrated bony union. They reported having 7 cases of superficial pin tract infections which were resolved with oral antibiotics. Tellisi, et al., describe applying a TSF for limb lengthening and ankle fusion simultaneously. ${ }^{4}$ The authors applied the TSF for ankle fusion and then brought patients back to the operating room for tibial lengthening. The surgeons performed the tibial osteotomy just distal to the tibial tuberosity. The authors also performed a fibular osteotomy to prevent tethering and angular deformity at the tibial lengthening site. The authors had 53 ankle fusions in which 12 underwent simultaneous tibial lengthening. $84 \%$ went on to complete fusion with two patients having significant non-unions (these patients were smokers). The average time in the fixator was 8.4 months with all patients having completely healed osteotomy sites. No significant pin tract infections were reported. 


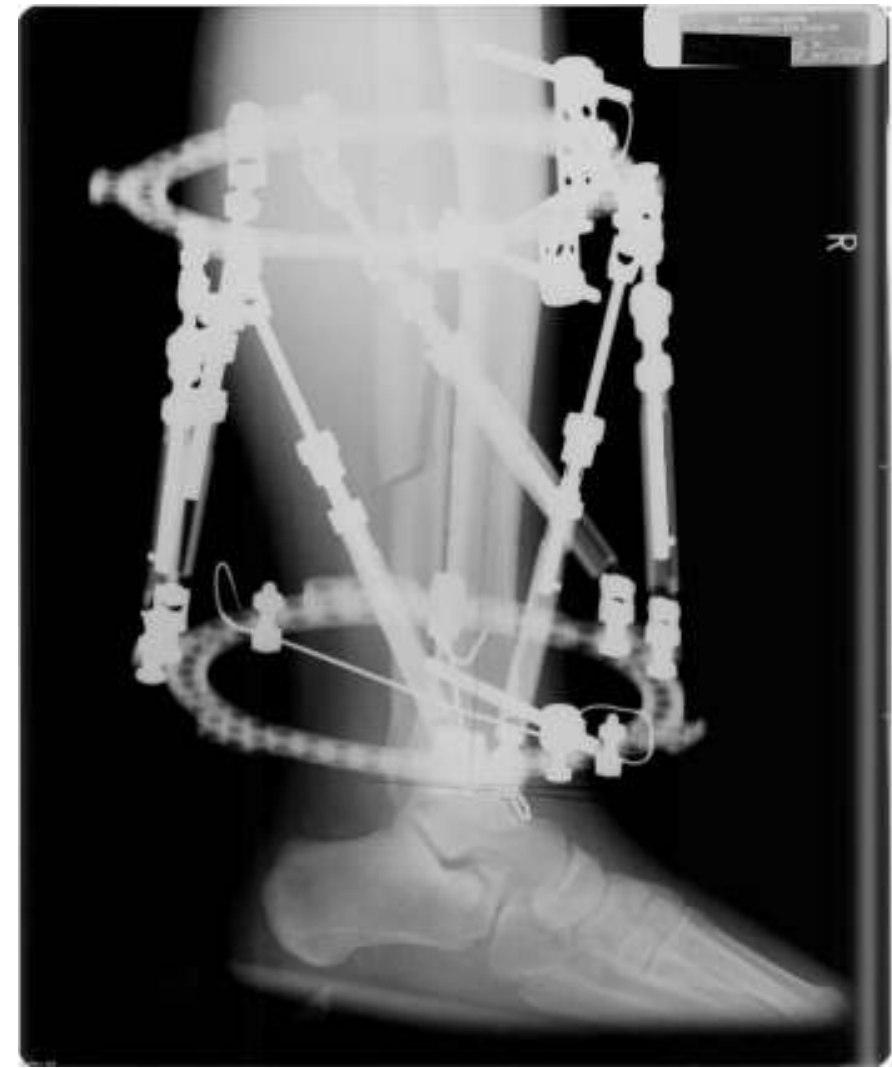

Figure 4 Immediate lateral post-operative radiograph showing fibular osteotomy.

A case study was reported by Mabit, et al., where a TSF was placed on a young girl with ankle varus that resulted from a malunited ankle fracture. ${ }^{5}$ The authors chose to correct her deformity gradually with a TSF preassembled and with the tibial rings oriented 30 degrees in the coronal plane matching the deformity. A tibial osteotomy was performed and distraction took place on the fourth post-operative day. The patient stayed in the TSF for 2.5 months. The patient went on to successful healing.

Feldman, et al., describe using the TSF for tibial malunions and nonunions. They had 18 patients in their study that had a TSF applied. The average time in the frame was 18.5 weeks. All patients went on to successful healing except one who developed a varus deformity through the healing fracture in the tibia.

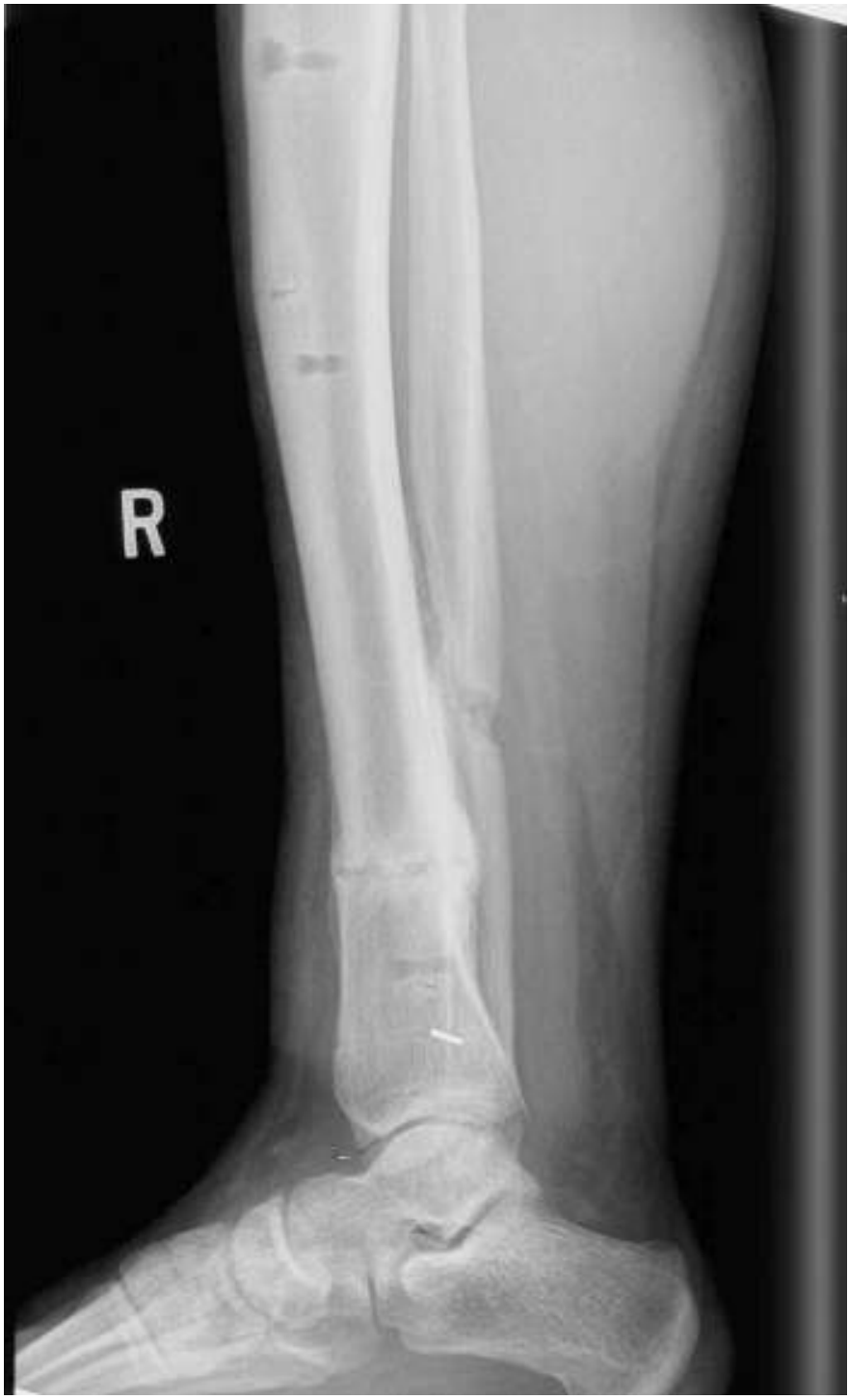

Figure 5 Final lateral post-operative radiograph showing healed osteotomy sites and decrease tibial procurvatum.

Fifteen of the 18 patients returned to preinjury activities at last follow-up. ${ }^{3}$ Matsubara, et al., describe application of a TSF for 3 patients due to ankle ankylosis. ${ }^{6} \quad$ All patients had limb length discrepancy and angulation deformity. The average time in the fixator was 216 days. All patients were able to walk normally with a plantigrade foot. 


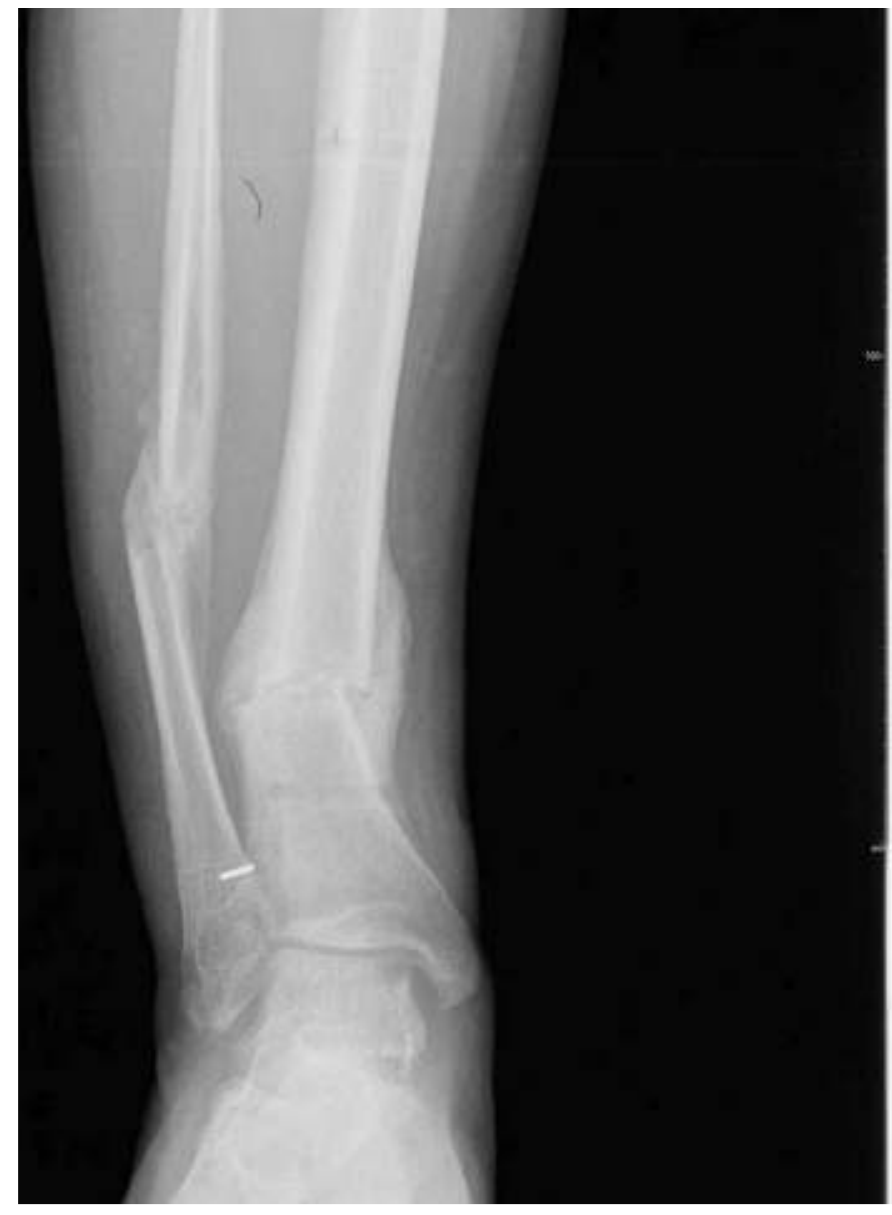

Figure 6 Final AP post-operative radiograph showing healed osteotomy sites with decrease in valgus position of the ankle.

\section{Conclusion}

The TSF is a useful tool for the surgeon to correct complex multiplane deformities of the lower extremity. Pre-operative parameters combined with the computer software make the TSF a simpler system as compared to traditional external fixators. There are various studies in the literature that demonstrate the usefulness and ease of this technique. Our patient was able to adjust the frame with ease and deformity correction was more precise using the computer software.

\section{References}

1. Chaudhary M. Taylor spatial frame-software-controlled fixator for deformity correction - The early Indian experience. Indian J Orthop 2007 41: 169-174.

2. Thiryayi WA, Naqui Z, Khan SA. Use of the Taylor Spatial Frame in Compression arthrodesis of the ankle: A study of 10 cases. J Foot Ankle Surg 2010 49: 182-187.

3. Feldman DS, Shin SS, Madan S, Koval KJ. Correction of tibial malunion and nonunion with six-axis analysis deformity correction using the Taylor Spatial Frame. J Orthop Trauma 2003 17: 549-554.

4. Tellisi N, Fragomen TA, Ilizarov S, Rozbruch SR. Limb salvage reconstruction of the ankle with fusion and simultaneous tibial lengthening using Ilizarov/Taylor Spatial Frame. Hospital Special Surgery, 2008 4:32-42.

5. Mabit C, Pecout C, Arnaud JP. Ilizarov's technique in correction of ankle malunion. J Orthop Trauma 1994 8: 520523.

6. Matsubara H, Tsuchiya H, Takato K, Tomita K. Correction of ankle ankylosis with deformity using the Taylor Spatial Frame: A report of three cases. Foot Ankle Int 2007 28: 1290-1294. 